\title{
Evaluation of FGFR1 as a diagnostic biomarker for ovarian cancer using TCGA and GEO datasets
}

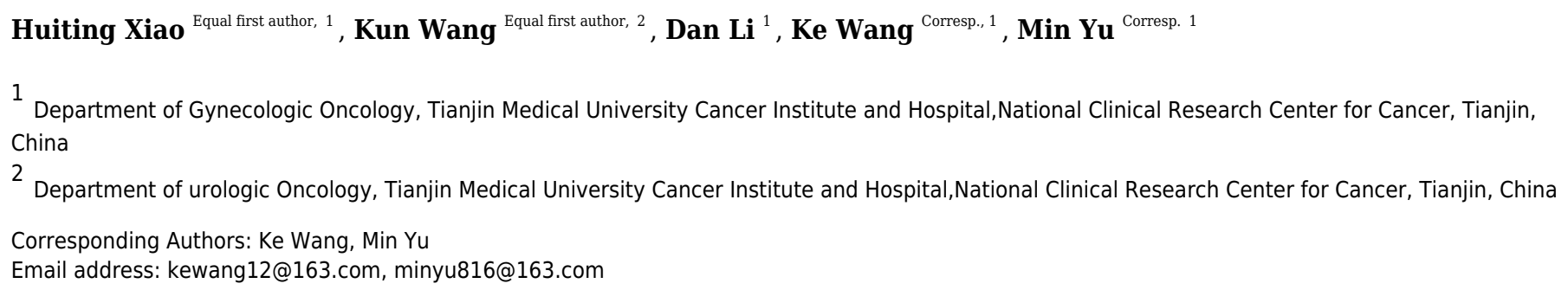

Background $\square$ Malignant ovarian cancer is associated with the highest mortality of all gynecological tumors. Designing therapeutic targets that are specific to OC tissue is important for optimizing $\mathrm{OC}$ therapies. This study aims to identify different expression patterns of genes related to FGFR1 and the usefulness of FGFR1 as diagnostic biomarker for OC. Methods $\square$ We collected data from The Cancer Genome Atlas (TCGA) and the Gene Expression Omnibus (GEO) databases. In the TCGA cohort we analyzed clinical information according to patient characteristics, including age, stage, grade, longest dimension of the tumor and the presence of a residual tumor. GEO data served as a validation set. We obtained data on differentially expressed genes (DEGs) from the two microarray datasets. We then used gene set enrichment analysis (GSEA) to analyze the DEG data in order to identify enriched pathways related to FGFR1. Results: Differential expression analysis revealed that FGFR1 was significantly downregulated in OC specimens. 303 patients were included in the TCGA cohort. The GEO dataset confirmed these findings using information on 75 Asian patients. The GSE105437 and GSE12470 database highlighted the significant diagnostic value of FGFR1 in identifying OC $\square A U C=1 \square p=0.0009$ and $A U C=0.8256$, $p=0.0015$ respectively). Conclusions: Our study examined existing TCGA and GEO datasets for novel factors associated with OC and identified FGFR1 as a potential diagnostic factor. Further investigation is warranted to characterize the role played by FGFR1 in OC. 
1 Evaluation of FGFR1 as a diagnostic biomarker for ovarian cancer using TCGA and GEO

2 datasets

3 Huiting Xiao ${ }^{\#, ~}$, Kun Wang ${ }^{\#, 2}$, Dan $\mathrm{Li}^{1}, \mathrm{Ke}$ Wang*, 1 , Min $\mathrm{Yu}^{*}, 1$

51 Department of Gynecologic Oncology, Tianjin Medical University Cancer Institute and Hospital,

6 National Clinical Research Center for Cancer, Tianjin 300060, P.R. China

72 Department of urologic Oncology, Tianjin Medical University Cancer Institute and Hospital,

8 National Clinical Research Center for Cancer, Tianjin 300060, P.R. China

\#Note: Huiting Xiao and Kun Wang contributed equally to this work.

Corresponding Authors: Ke Wang, Min Yu

Emails address: kewang12@163.com,minyu816@163.com

\section{Abstract}

Background:Malignant ovarian cancer is associated with the highest mortality of all gynecological tumors. Designing therapeutic targets that are specific to OC tissue is important for optimizing OC therapies. This study aims to identify different expression patterns of genes related to FGFR1 and the usefulness of FGFR1 as diagnostic biomarker for OC.

Methods: We collected data from The Cancer Genome Atlas (TCGA) and the Gene Expression Omnibus (GEO) databases. In the TCGA cohort we analyzed clinical information according to patient characteristics, including age, stage, grade, longest dimension of the tumor and the presence of a residual tumor. GEO data served as a validation set. We obtained data on differentially expressed genes (DEGs) from the two microarray datasets. We then used gene set enrichment analysis (GSEA) to analyze the DEG data in order to identify enriched pathways related to FGFR1.

Results: Differential expression analysis revealed that FGFR1 was significantly downregulated in

OC specimens. 303 patients were included in the TCGA cohort. The GEO dataset confirmed these 
findings using information on 75 Asian patients. The GSE105437 and GSE12470 database highlighted the significant diagnostic value of FGFR1 in identifying OC (AUC $=1, p=0.0009$ and $\mathrm{AUC}=0.8256, \mathrm{p}=0.0015$ respectively).

Conclusions: Our study examined existing TCGA and GEO datasets for novel factors associated with OC and identified FGFR1 as a potential diagnostic factor. Further investigation is warranted to characterize the role played by FGFR1 in OC.

Keywords: Ovarian cancer, Targeted treatment, FGFR1, RNA-sequencing

\section{Introduction}

Ovarian cancer is a common gynecological malignant cancer. Because its mortality rate ranks first among gynecological tumors, it has become a major health risk for women (Chen S, 2018; Meys EMJ, 2018). The onset of ovarian cancer is usually hidden and most patients have no obvious symptoms in the early stages (Partridge EE, 1996). Therefore, at the time of diagnosis patients may have already reached relatively advanced stages of OC. The five-year survival rate of patients is approximately $40 \%$ (Partridge EE, 1996; Stewart SL, 2017) while the overall five-year survival rate for patients whose cancer has reach an advanced stage is only $20-30 \%$ (Bhatt P, 2016). The ovarian cancer patients who received guideline-based treatment have better five-year survival rate. However, even after initial treatment, most patients with epithelial ovarian cancer will experience a recurrence, and currently there is no cure (Beltrame L, 2015; Bruchim I, 2016; Ganapathi MK, 2016). Moreover, traditional treatment methods, including surgery and chemotherapy, do not significantly affect the survival rate of the disease. In consequence, the development of new methods to detect $\mathrm{OC}$ in the early stages of its progression as well as individualized treatment plans for ovarian cancer are key steps for improving clinical efficacy and safety.

The targeted treatment of OC plays an increasingly important role in the comprehensive treatment of $\mathrm{OC}$ and new tumor treatment strategies depend on the search for new targets (Huang Z, 2018; Shivange G 2018; Villar-Prados A, 2018; Zhai J LR, 2018). With the development of sequencing technology and acquirement of a large amount of biological data, bioinformatics 
55

56

57

can be used to understand and find new biomarkers of tumor (Dingquan Yang, 2020; Guang Wu, 2019; Tao Lu, 2019). The Vascular Endothelial Growth Factor (VEGF) signaling pathway is essential for tumor angiogenesis (Albini A BA, 2018; Caron-Beaudoin É VR, 2018; Lee DH LM, 2018; Lin R, 2018). Anti VEGF therapies clearly display anti-angiogenic efficacy in the treatment of pathological angiogenesis as well as cancers including lung cancer (Kabbinavar F 2014), glioma (Griveau A, 2018), metastatic renal cell carcinoma (Escudier B, 2007; Grünwald V SC, 2011), metastatic colorectal cancer (Bennouna J, 2018), and ovarian cancer(González Martín A OA, 2018; Lee JY, 2018). Clinical data has shown that current anti-angiogenic targeted drugs have been successfully introduced in the anti-cancer therapy, but they still have more drawbacks (Abdullah S E 2012; Poletto V, 2018; Wang S, 2018). VEGF is a crucial angiogenic factor. A lot of angiogenic factors, including angiopoietins, platelet derived growth factor (PDGF) and fibroblast growth factor (FGF), collaborate with VEGF in the angiogenic process (Abdullah S E 2012; Ip CKM, 2018; Lieu C, 2011; Semrad TJ and Mack PC, 2011).

The human fibroblast growth factor receptor (FGFR) family consists of four members: FGFR1 to FGFR4 and the native ligand of FGFRs is fibroblast growth factors (FGFs) (Lemmon M.A., 2010; Weiner H.L., 2000). Dysregulation of FGFRs has been implicated in a wide variety of cancers, such as urothelial carcinoma, hepatocellular carcinoma, ovarian cancer and lung adenocarcinoma (Cole C, 2010; Fearon A E, 2013; Shuyan D, 2019; ). There is evidence that differential expression in specific FGFR may be related to prognosis or sensitivity to cancer treatments (Turner N, 2010). At this time, several FGFR inhibitors are FDA approved for treatment of cancer, including lenvatinib approved for iodine-refractory, well-differentiated thyroid carcinoma, regorafenib approved for advanced colorectal carcinoma and drug-resistant gastrointestinal stromal tumors (GIST), ponatinib approved for drug-resistant chronic myelogenous leukemia (CML) and Philadelphia chromosome-positive acute lymphocytic leukemia (ALL), and pazopanib approved for renal cell carcinoma and sarcoma. The FGF/FGFRsystem plays a critical role in carcinogenesis, but little is known of its influence in ovarian cancer. This study aims to provide insight into the differential expression of FGFR-linked genes in 
82

OC and normal tissues by comparing TCGA and GEO data. The detection of DEGs between OC and non-OC tissues may facilitate the identification of novel therapeutic targets of OC. To better understand the role of FGFR1 in ovarian cancer in individual studies, a meta-analysis was designed to synthesize current findings. Here, DEGs found by comparing gene expression in OC and nonOC samples were screened using the R software. The enrichment pathway analysis of DEGs was performed using GSEA. Our results suggest that the significant diagnostic ability of FGFR1, but also note that more evidence is needed to improve disease prognosis and to design effective drugs to treat ovarian cancer.

\section{Methods}

\subsection{TCGA data description}

To obtain a dataset based on studies listed in The Cancer Gene Atlas (TCGA) database, patients' gene expression and clinical information were downloaded from the publicly available TCGA Data Portal at https://tcga-data.nci.nih.gov/tcga/. For the TCGA cohort, gene expression profiles were studied in 303 female patients with histologically confirmed ovarian cancer who had undergone prior surgical resection and received no pretreatment. Gene expression profiles were measured experimentally using an Illumina HiSeq2000 RNA Sequencing instrument at the University of North Carolina TCGA genome characterization center. Level 3 data was downloaded from the TCGA data coordination center. This dataset shows gene-level transcription estimates, as in $\log 2(\mathrm{x}+1)$ transformed RSEM normalized counts. Genes were mapped onto the human genome coordinates using HUGO probeMap. Reference to method description from University of North Carolina TCGA genome characterization center. All analyses were performed using FDR (False Discovery Rate) corrected q-values adjusted to 0.05 .

\subsection{GEO dataset selection}

We obtained OC microarray profiles from the GEO database (http://www.ncbi.nlm.nih.gov/geo/). The following keywords were used to query the GEO 
database: (ovary OR ovarium OR oophoron OR ootheca OR germarium) AND (cancer OR carcinoma OR tumor OR tumour OR neoplas* OR malignan*). All the functional genomics data of FGFR1 were requested and assembled from the GEO Database (http://www.ncbi.nlm.nih.gov/geo/) with the closing date of 10 February 2019. The inclusion and exclusion criteria of this study were as follows: (1) only human tissue studies were included, and samples from cell lines and animal models were excluded; (2) the expression data of the experimental and control groups must be provided or calculated; (3)dual-channel microarray studies were excluded; (4)DNA methylation arrays studies were excluded; (5)studies without a control group, as well as studies on other cancers were excluded; (6) studies with less than 20 cases were excluded. Differentially expressed genes (DEGs) identified by comparing expression in ovarian cancer and normal samples were analyzed using the R language software. Samples with both corrected P-values $<0.05$ and log fold changes $(\mathrm{FC})>1$ were deemed to be DEGs.

\subsection{Gene set enrichment analysis (GSEA)}

GSEA was performed using GSEA software 3.0 from the Broad Institute as previously described (Subramanian A, 2005). GSEA evaluates gene expression profiles from samples belonging to normal and tumorous samples, and analyzes data in terms of gene sets. Normalized enrichment scores (NES) were acquired by analyzing genes with permutations 1000 times. A gene set was considered to be significantly enriched if it had a normal $p$-value $<0.05$. However, if the number of samples per group was fewer than 7, gene set was selected as permutation type, and FDR $<0.05$ was set as the criterion of statistical significance.

\subsection{Statistical analysis}

Overall survival was calculated from the data of surgery to the date of death or last followup. Recurrence-free survival was defined as the time from the date of surgery to the date of first recurrence or last follow-up. Patients without events or death were recorded as censored at the time of last follow-up. Pathological stage and grade were considered to be distinct categorical variables. 
136

137

138

139

140

141

142

143

144

145

146

147

148

149

150

151

152

153

154

155

156

157

158

159

160

161

162

Age, longest dimension and residual tumor were included in the model as continuous variables. Gene expression levels from the TCGA database were included as continuous variables.

All data are displayed as mean \pm standard deviation (SD) for each group. Student's $t$-tests were performed to test for differences in mean values of vaviables of interest between two groups, whereas one-way analyses of variance (ANOVAs) were used to test for differences in means among three or more groups. Standardized mean difference (SMD) was applied to evaluate the association between FGFR1 levels and OC using RevMan 5.3.0. We pooled SMDs across GEO datasets using the Mantel-Haenszel formula (fixed-effect model) or the DerSimonian-Laird formula (random-effect model). A fixed-effect model was adopted when the Q statistic was considered significant ( $\mathrm{p}>0.1$, or $\mathrm{I}^{2}<50 \%$ ); otherwise, a random-effect model was used.

\section{Results}

\subsection{Study Characteristics}

The present study consists of several processes sequentially (Fig. 1), that is, TCGA-based RNA-seq data aggregation and clinical values, GEO-based data verification, meta-analyses based on GEO and TCGA, and multiple bioinformatics analyses. A total of twelve GEO datasets (GSE105437, GSE66957, GSE66387, GSE40595, GSE29450, GSE29156, GSE27651, GSE26712, GSE18521, GSE18520, GSE17308, and GSE12470) were collected for use in our study. Two datasets (GSE105437 and GSE12470) contained detailed information on Asian populations, and these were used in this study. The platform for GSE105437 was GPL570, the [HG-U133_Plus_2] Affymetrix Human Genome U133 Plus 2.0 Array, includes 5 normal ovarian surface epithelial samples and 10 high-grade stage III invasive serous ovarian cancer samples. The platform for GSE12470 was GPL887, the Agilent-012097 Human 1A Microarray (V2) G4110B; this platform, included 10 normal peritoneum samples, 35 advanced stage serous ovarian cancer samples and 8 early stage serous ovarian cancer samples. In addition, 308 cases with EGFR or FGFR expression data were screened in TCGA database excluding 5 cases with recurrence, and 303 patients form this database were included in this study. A flowchart and other detailed 
information regarding the studies included in the meta-analysis are shown in Fig. 1.

\subsection{FGFR expression in OC based on GEO}

FGFR or EGFR expression was initially assessed in a series of ovarian tumor and normal tissues based on GEO dataset (Figure 2). The datasets were analyzed with GeneSpringGX and mapped with Graphpad 6.0 software. The expression levels of FGFR1 in ovarian cancer tissues were significantly lower than in non-cancer control tissues in both GSE105437 and GSE12470 with analyses performing Fold change cut-off adjusted to 2.0 and corrected p-values cut-off adjusted to $0.05(\mathrm{p}<0.0001$ and $\mathrm{p}=0.0032$, respectively; Figure $2 \mathrm{~A}$ and $2 \mathrm{~B})$. The expression of FGFR1 was significantly different in advanced ovarian cancer, but not in early ovarian cancer in GSE12470 ( $\mathrm{p}=0.0124$; Figure 2C). The expression levels of EGFR in ovarian cancer tissues were significantly lower than in non-cancer control tissues in GSE105437 ( $p=0.0008$; Figure 2D), whereas no difference was found in GSE12470. The expression levels of FGFR2 and FGFR3 were significantly different only in GSE12470 $(\mathrm{p}=0.0046$ and $\mathrm{p}=0.0049$, respectively; Figure $2 \mathrm{E}$ and 2F). Therefore, FGFR1 was chosen for subsequent analysis.

To have a more comprehensive understanding of the biomedical predictive value of FGFR1, ROC Curves were provided to investigate the diagnostic value of GSE105437 and GSE12470 in distinguishing OC tissues from normal controls. As shown in Fig. 3, the Area Under the Curve (AUC) of GSE105437 outperformed GSE12470 (AUC=1, $\mathrm{p}=0.0009$ and $\mathrm{AUC}=0.8256, \mathrm{p}=0.0015$ respectively). Therefore, we considered FGFR1 might play an important role in diagnosing ovarian cancer. In brief, no significant differences were found between OC and nontumor groups based on the GSE105437 and GSE12470 data considered here (SMD=-6.22; 95\% CI,-7.48 to $-4.96 ; \mathrm{p}=0.93$, Fig. 4A). Moreover, we found no significant heterogeneity by fixed-effected model $(\mathrm{p}<0.00001$, $\mathrm{I}^{2}=0 \%$ ). Finally, the funnel plot shown in Fig.4B did not imply significant publication bias.

\subsection{Identification of DEGs in ovarian cancer using integrated bioinformatics}

The two ovarian cancer gene expression microarray datasets GSE105437 and GSE12470 
were analyzed using the R limma package and were sorted according to logfold-change values with corrected $p$-values $<0.05$. The results of DEGs from the two databases showed in Fig.5, and FGFR was identified both in the down-regulated genes (Green points).

\subsection{GSEA enrichment analysis of DEGs}

To find enriched pathways related to FGFR1 and to identify its potential function, we performed a GSEA (Fig.6). First, common pathways were found by comparing the GSE105437 (Fig.6 A) and GSE12470 (Fig.6 B) datasets. Although, many of these common pathways were not significantly associated with FGFR1, we found that the KEGG adherent junction signaling pathway was significantly correlated with FGFR1 in the GSE105437 dataset (Fig.6 C).

\subsection{Clinical characteristics of patients from the TCGA}

The TCGA cohort consisted of 303 female patients with histologically confirmed ovarian cancer who had undergone prior surgical resection and received no pretreatment. Summary data of clinical indicators such as age, stage, grade, the longest dimension of the tumor, ANS, and residual tumor were shown in Table 1 . We tested whether these variables were correlated with the relative expression of FGFR1 mRNA in OC tissues. We found no significant differences in the clinical features for all the tested parameters. The median follow-up time was 26.11 months and 182 patients died during follow-up. The results of the overall survival (OS) analyses, as calculated using the Kaplan-Meier method are shown in Table 2 and Fig. 7; we found significant differences in survival between all groups. We combined stage I and II into a single group, as well as grades G1 and G2 into another group for statistical analysis, since only 1 patient was included in the dataset for both stage I and grade G1. Residual tumors were found to be independently associated with increased OS according to univariate analysis $(\mathrm{p}=0.001)$. In contrast, we found no statistical evidence that other characteristics, including age, stage, grade, tumor longest dimension, and ANS were independent prognostic factors. In addition, our results showed that patients with larger residual tumors, high FGFR expression, and advanced cancer stages were associated with shorter 
217

218

219

220

221

222

223

224

225

226

227

228

229

230

231

232

233

234

235

236

237

238

239

240

241

242

243

$\mathrm{OS}(\mathrm{p}=0.001, \mathrm{p}=0.233, \mathrm{p}=0.148$, respectively; Fig. 7$)$.

\section{Discussion}

Ovarian cancer is the deadliest gynecological malignant tumor due to the fact that this cancer is associated with delayed diagnosis, recurrence, and metastasis (Chen S, 2018; Meys EMJ, 2018; Partridge EE, 1996). Targeted treatments and immunohistochemistry offer the hope of improved treatments for OC patients in the future. Moreover, identifying biomarkers for targeted treatments is important for effective cancer diagnosis and treatment (Shivange G 2018; Villar-Prados A, 2018). RNA sequencing is an accurate method used to identify such biomarkers (Coenen-Stass AML, 2018; Jiang K, 2018). Thus, RNA-sequencing will likely become more important during patient treatment, both for predicting the efficacy of different therapies as well as in monitoring toxicity.

FGFR is a promising cancer biomarkers and has a canonical tyrosine kinase receptor structure (Lieu C, 2011; Semrad TJ and Mack PC, 2011). The FDFR family contains four members, FGFR1, FGFR2, FGFR3, and FGFR4 (Eswarakumar VP, 2005). FGFR signaling is initiated by interaction with 23 different FGF ligands, and is involved in many cellular processes, including proliferation, differentiation, migration, survival, organogenesis, angiogenesis, and embryonic development (Eswarakumar VP, 2005; L, 1999; N, 2015; Touat M, 2015). Recently, several mutations and alterations in FGFRs have been reported in cancers. In addition, specific alterations of FGFR have been found to be more frequent in certain types of tumors, thus making FGFR a suitable biomarker (Brooks AN, 2012; N, 2015). However, the relationship between FGFR1 and ovarian cancer has not been well determined.

In this study, we examined FGFR1 expression in OC by examining RNA expression profiles in cancerous tissues sourced from the TCGA and GEO datasets. In samples from the TCGA database, we identified the clinical characteristics of OC patients that were found to be associated with FGFR1 expression. However we were not able to draw a strong functional link between FGFR1 and OC, since the TCGA dataset contained too fewer noncancerous samples, thus reducing 
244 the statistical power of the analysis. To gain insight into the functional molecular pathways of 245 FGFR1 that are implicated in the development and progression of ovarian cancer, two samples 246 from the GEO database (datasets GSE105437 and GSE12470, containing 53 OC samples and 15 247 non-OC samples) were subjected to further examination. No clinical data was available for these 248 datasets, and therefore we were not able to corroborate our previous finding. Next, we investigated 249 the expression patterns of FGFR1 in ovarian cancer samples from both datasets. Survival analysis 250 showed that low expression of FGFR1 was closely associated with poor OS. Moreover, using data 251 from the GEO datasets, we also found that FGFR1 was downregulated in ovarian cancer samples relative to normal tissues. However, no evidence indicated that FGFR1 is an independent factor affecting the clinical outcomes of ovarian cancer patients. Taken together, the results of our investigation, we found that FGFR1 might have significant diagnostic value in predicting OC for Asian populations (i.e. for the GSE105437 dataset: $\mathrm{AUC}=1, \mathrm{p}=0.0009$ and GSE12470 dataset: $\mathrm{AUC}=0.8256, \mathrm{p}=0.0015)$. These results suggest that FGFR1 may be a very good diagnostic biomarker but not a good prognosis marker. More samples should be examined to validate these 258 findings.

\section{Conclusions}

In conclusion, this is a preliminary study designed to investigate the role played by FGFR1 in ovarian cancer. The meta analyses of ovarian cancer that we report here significantly extends our knowledge base to cognize that FGFR1 may be a good diagnostic biomarker in Asian populations (Fig.3). In the future, these findings should be validated using new datasets, and immunohistochemistry or western blotting should be used to confirm transcriptomic results by examining protein contents. Finally, research into the value of FGFR1 in cancer diagnosis should be performed in order to assess its usefulness for potential clinical application.

\section{Conflict of interest}

270 None declared. 


\section{Acknowledgments}

273 This work was supported by the National Natural Science Foundation of China (NO. 81602299),

274 Tianjin Health and Family Planning Commission Foundation of Science and Technology (NO.

16KG129) and National Natural Science Foundation of China (NO. 81602245).

\section{References}

Abdullah S E P-SR. 2012. Mechanisms of resistance to vascular endothelial growth factor blockade. Cancer 118. DOI: $10.1002 /$ cncr.26540

Albini A BA ND, Mortara L. 2018. Contribution to Tumor Angiogenesis From Innate Immune Cells Within the Tumor Microenvironment: Implications for Immunotherapy. Frontiers in Immunology 9. DOI: 10.3389/fimmu.2018.00527

Beltrame L DMM, Fruscio R, Calura E, Chapman B, Clivio L, Sina F, Mele C, Iatropoulos P, Grassi T, Fotia V, Romualdi C, Martini P, Noris M, Paracchini L, Craparotta I, Petrillo M, Milani R, Perego P, Ravaggi A, Zambelli A, Ronchetti E, D'Incalci M and Marchini S. 2015. Profiling cancer gene mutations in longitudinal epithelial ovarian cancer biopsies by targeted next-generation sequencing: a retrospective study. Annals of Oncology Official Journal of the European Society for Medical Oncology 26:1363-1371. DOI: 10.1093/annonc/mdv164

Bennouna J HS, Bertaut A, Bouche O, Deplanque G, Borel C, Francois E, Conroy T, Ghiringhelli F, des Guetz G, Seitz JF, Artru P, Hebbar M, Stanbury T, Denis MG, Adenis A and Borg C. 2018. Continuation of Bevacizumab vs Cetuximab Plus Chemotherapy After First Progression in KRAS Wild-Type Metastatic Colorectal Cancer: The UNICANCER PRODIGE18 Randomized Clinical Trial. JAMA Oncol. DOI: 10.1001/jamaoncol.2018.4465

Bhatt P VI, Patil S, Amrutiya J, Bhattacharya C, Misra A and Mashru R. 2016. Role of antibodies in diagnosis and treatment of ovarian cancer: Basic approach and clinical status. $J$ Control Release 226:148-167. DOI: 10.1016/j.jconrel.2016.02.008

Brooks AN KEaSP. 2012. Molecular pathways: fibroblast growth factor signaling: a new therapeutic opportunity in cancer. Clinical Cancer Research 18:1855-1862. DOI: 10.1158/1078-0432.CCR-11-0699

Bruchim I WN, Alpert Y, Sade D, Piura E and Fishman A. 2016. High Efficacy and Low Toxicity of the Modified Docetaxel and Carboplatin Protocol in Patients with Recurrent Ovarian Cancer-A Phase 2 Cohort Study. International journal of gynecological cancer 26:640-647. DOI: 10.1097/igc.0000000000000666

Caron-Beaudoin É $\quad$ VR SJ. 2018. Effects of Neonicotinoid Pesticides on Promoter-Specific Aromatase (CYP19) Expression in Hs578t Breast Cancer Cells and the Role of the VEGF Pathway. Environmental Health Perspectives 126. DOI: 10.1289/EHP2698

Chen S WL, Sun KX, Liu Y, Guan X, Zong ZH and Zhao Y. 2018. LncRNA PCGEM1 Induces Ovarian Carcinoma Tumorigenesis and Progression Through RhoA Pathway. Cell Physiol Biochem 47:1578-1588. DOI: $10.1159 / 000490931$

Coenen-Stass AML SH, Gatto S, Godfrey C, Bhomra A, Krjutskov K, Hart JR, Westholm JO, O'Donovan L, Roos A, Lochmuller H, Puri PL, El Andaloussi S, Wood MJA and Roberts TC. 2018. Comprehensive RNA- 
310

311

312

313

314

315

316

317

318

319

320

321

322

323

324

325

326

327

328

329

330

331

332

333

334

335

336

337

338

339

340

341

342

343

344

345

346

347

348

349

350

Sequencing Analysis in Serum and Muscle Reveals Novel Small RNA Signatures with Biomarker Potential for DMD. Molecular Therapy Nucleic Acids 13: 1-15. DOI: 10.1016/j.omtn.2018.08.005

Cole C, Lau S, Backen A, Clamp A, Rushton G, Dive C, Hodgkinson C, Mcvey R Kitchener H and Jayson GC. 2010. Inhibition of FGFR2 and FGFR1 increases cisplatin sensitivity in ovarian cancer. Cancer biology \& therapy 10(5):495-504. DOI: 10.4161/cbt.10.5.12585

Dingquan Yang YJ, Yanqing Li and Xuedong Fang. 2020. Clinical characteristics and prognostic value of MEX3A mRNA in liver cancer. PeerJ 8:e8252. DOI 10.7717/peerj.8252

Escudier B PA, Koralewski P, Ravaud A, Bracarda S, Szczylik C, Chevreau C, Filipek M, Melichar B, Bajetta E, Gorbunova V, Bay JO, Bodrogi I, Jagiello-Gruszfeld A, Moore N and investigators AT. 2007. Bevacizumab plus interferon alfa-2a for treatment of metastatic renal cell carcinoma: a randomised, double-blind phase III trial. Lancet 370:2103-2111. DOI: 10.1016/s0140-6736(07)61904-7

Eswarakumar VP LIaSJ. 2005. Cellular signaling by fibroblast growth factor receptors. Cytokine Growth Factor Rev 16:139-149. DOI : $10.1016 /$ j.cytogfr.2005.01.001

Fearon A E, Gould C R, Grose R P. 2013. FGFR signalling in women's cancers. International Journal of Biochemistry \& Cell Biology 45(12):2832-2842. DOI: 10.1016/j.biocel.2013.09.017

Ganapathi MK JW, Sehouli J, Michener CM, Braicu IE, Norris EJ, Biscotti CV, Vaziri SA and Ganapathi RN. 2016. Expression profile of COL2A1 and the pseudogene SLC6A10P predicts tumor recurrence in high-grade serous ovarian cancer. International Journal of Cancer 138:679-688. DOI: 10.1002/ijc.29815

González Martín A OA EA, Pfisterer J ,Ledermann JA ,Kristensen G,Bertrand MA 8 ,Beale P 9 ,CervantesA 10 ,Kent E 11 ,Kaplan RS 12 ,Parmar MKB 13 ,Scotto N 14 ,Perren TJ;ICON7 investigators. 2018. Exploratory outcome analyses according to stage and/or residual disease in the ICON7 trial of carboplatin and paclitaxel with or without bevacizumab for newly diagnosed ovarian cancer. DOI: $10.1016 /$ j.ygyno.2018.08.036

Grünwald V SC FM, Ganser A ,Busch J,Weikert S. 2011. Treatment of everolimus-resistant metastatic renal cell carcinoma with VEGF-targeted therapies. British Journal of Cancer 105:1635-1639. DOI: 10.1038/bjc.2011.389

Griveau A SG, Shelton SJ, Kupp R, Jahangiri A, Obernier K, Krishnan S, Lindberg OR, Yuen TJ, Tien AC, Sabo JK, Wang N, Chen I, Kloepper J, Larrouquere L, Ghosh M, Tirosh I, Huillard E, Alvarez-Buylla A, Oldham MC, Persson AI, Weiss WA, Batchelor TT, Stemmer-Rachamimov A, Suva ML, Phillips JJ, Aghi MK, Mehta S, Jain RK and Rowitch DH. 2018. A Glial Signature and Wnt7 Signaling Regulate Glioma-Vascular Interactions and Tumor Microenvironment. Cancer Cell 33. DOI: 10.1016/j.ccell.2018.03.020

Guang Wu FW, Kai Li, Shugen Li, Chunchun Zhao, Caibin Fan and Jianqing Wang. 2019. Significance of TP53 mutation in bladder cancer disease progression and drug selection. PeerJ 7:e8261. DOI: 10.7717/peerj.8261

Huang Z ZW, Li Y, Cao M, Wang T, Ma Y, Guo Q, Wang X, Zhang C, Zhang C, Shen W, Liu Y, Chen Y, Zheng J, Yang S, Fan Y and Xiang R. 2018. Novel hybrid molecule overcomes the limited response of solid tumours to HDAC inhibitors via suppressing JAK1-STAT3-BCL2 signalling. Theranostics 8:4995-5011. DOI: $10.7150 /$ thno. 26627

Ip CKM NP, Jeong KJ, Shao SH, Ju Z, Leonard PG, Hua X, Vellano CP, Woessner R, Sahni N, Scott KL and Mills GB. 2018. Neomorphic PDGFRA extracellular domain driver mutations are resistant to PDGFRA targeted therapies. Nature Communications 9. DOI: 10.1038/s41467-018-06949-w

Jiang K PK, Wong L, Chen Y, Borowitz D, Goetz D, Sheehan D, Frederick C, Tutino VM, Meng H and Jarvis JN. 2018. RNA sequencing data from neutrophils of patients with cystic fibrosis reveals potential for developing 
351

352

353

354

355

356

357

358

359

360

361

362

363

364

365

366

367

368

369

370

371

372

373

374

375

376

377

378

379

380

381

382

383

384

385

386

387

388

389

390

391

biomarkers for pulmonary exacerbations. Journal of Cystic Fibrosis. DOI: 10.1016/j.jcf.2018.05.014

Kabbinavar F FL, Hainsworth J , Kasubhai S and Kressel B MT, Patel T ,Rubin M ,White L ,Yang JC ,Klughammer B ,Colburn D ,Miller V ,JohnsonB. 2014. Biomarker analyses from a randomized, placebocontrolled, phase IIIb trial comparing bevacizumab with or without erlotinib as maintenance therapy for the treatment of advanced non-small-cell lung cancer (ATLAS). $J$ Thorac Oncol. DOI: 10.1097/JTO.0000000000000274

L KPaC-W. 1999. Signal transduction by fibroblast growth factor receptors. Frontiers in bioscience : a journal and virtual library 4:D165. DOI: 10.2741/Klint

Lee DH LM SY, Hong HJ ,An HJ ,Kang JS ,Kim HM. 2018. Multi-paratopic VEGF decoy receptor have superior anti-tumor effects through anti-EGFRs and targeted anti-angiogenic activities. Biomaterials 171:34-45. DOI: 10.1016/j.biomaterials.2018.04.029

Lee JY PJ, Park SY, Lee JW, Kim JW, Kim YB, Jeong DH, Lee KB, Kim TH, Lee IH, Choi MC, Kim KH, Kim YM, Lee YJ, Kang S, Investigators K and Pujade-Lauraine E. 2018. Real-world effectiveness of bevacizumab based on AURELIA in platinum-resistant recurrent ovarian cancer (REBECA): A Korean Gynecologic Oncology Group study (KGOG 3041). DOI: 10.1016/j.ygyno.2018.10.031

Lemmon M.A. SJ. 2010. Cell signaling by receptor tyrosine kinases. Cell 141:1117-1134. DOI: 10.1016/j.cell.2010.06.011

Lieu C HJ, Overman M, Tran H and Kopetz S. 2011. Beyond VEGF: inhibition of the fibroblast growth factor pathway and antiangiogenesis. Clinical Cancer Research 17:6130-6139. DOI: 10.1158/1078-0432.CCR-11-0659

Lin R HJ, Wang L, Li Y, Lipowska M, Wu H, Yang J and Mao H. 2018. Bevacizumab and near infrared probe conjugated iron oxide nanoparticles for vascular endothelial growth factor targeted MR and optical imaging. Biomater Sci 6:1517-1525. DOI: 10.1039/C8BM00225H

Meys EMJ JL, Ramaekers BLT, Dirksen CD, Kooreman LFS, Slangen BFM, Kruitwagen R and Van Gorp T. 2018. Economic evaluation of an expert examiner and different ultrasound models in the diagnosis of ovarian cancer. European Journal of Cancer 100. DOI: 10.1016/j.ejca.2018.05.003

N ODaI. 2015. The Fibroblast Growth Factor signaling pathway. Wiley interdisciplinary reviews Developmental biology 4:215-266. DOI: 10.1002/wdev.176

Partridge EE PJaMH. 1996. The National Cancer Data Base report on ovarian cancer treatment in United States hospitals. Cancer 78: 2236-2246. DOI: 10.1002/(sici)1097-0142(19961115)78:10<2236::aidcncr28>3.0.co;2

Poletto V RV, Biggiogera M, Guerra G, Moccia F and Porta C. 2018. The role of endothelial colony forming cells in kidney cancer's pathogenesis, and in resistance to anti-VEGFR agents and mTOR inhibitors: A speculative review. Critical Reviews in Oncology/hematology 132:89-99. DOI: 10.1016/j.critrevonc.2018.09.005

Semrad TJ and Mack PC. 2011. Fibroblast growth factor signaling in non-small-cell lung cancer. Clinical Lung Cancer 13:90-95. DOI: 10.1016/j.cllc.2011.08.001

Shivange G UK, Przanowski P , Perry JSA, Jones J , HaggartR , Kostka C , Patki T , Stelow E , Petrova Y , Llaneza D , MayoM ，Ravichandran KS , Landen CN , Bhatnagar S and J T-S. 2018. A Single-Agent Dual-Specificity Targeting of FOLR1 and DR5 as an Effective Strategy for Ovarian Cancer. Cancer Cell 34:331-345. DOI: 10.1016/j.ccell.2018.07.005

Shuyan D, Zhan Z, Zhuchu C, Guangyu X and Yongheng C. 2019. Fibroblast Growth Factor Receptors (FGFRs): Structures and Small Molecule Inhibitors. Cells 8(6): 614. DOI: 10.3390/cells8060614 
392

393

394

395

396

397

398

399

400

401

402

403

404

405

406

407

408

409

410

411

412

413

414

415

416

417

418

419

420

421

422

423

424

425

426

427

428

429
Stewart SL HR, Matz M, Rim SH, Sabatino SA, Ward KC and Weir HK. 2017. Disparities in ovarian cancer survival in the United States (2001-2009): Findings from the CONCORD-2 study. Cancer 123:5138-5159. DOI: 10.1002/cncr.31027

Subramanian A TP, Mootha VK, Mukherjee S, Ebert BL, Gillette MA, Paulovich A, Pomeroy SL, Golub TR, Lander ES and Mesirov JP. 2005. Gene set enrichment analysis: a knowledge-based approach for interpreting genome-wide expression profiles. Proceedings of the National Academy of Sciences of the United States of America 102:15545-15550.

Tao Lu SC, Le Qu, Yunlin Wang, Hong-duo Chen and Chundi He. 2019. Identification of a five-miRNA signature predicting survival in cutaneous melanoma cancer patients. PeerJ 7:e7831. DOI: 10.7717/peerj.7831

Touat M IE, Postel-Vinay S, Andre F and Soria JC. 2015. Targeting FGFR Signaling in Cancer. Clinical Cancer Res 21:2684-2694. DOI: 10.1158/1078-0432.CCR-14-2329

Turner N PA, Sharpe R, Lambros M, Geyer F, Lopez-Garcia MA, Natrajan R, Marchio C, Iorns E, Mackay A, Gillett C, Grigoriadis A, Tutt A, Reis-Filho JS, Ashworth A. 2010. FGFR1 amplification drives endocrine therapy resistance and is a therapeutic target in breast cancer. Cancer Research 70:2085-2094. DOI: 10.1158/00085472.CAN-09-3746

Villar-Prados A WS, Court KA, Ma S, LaFargue C, Chowdhury MA, Engelhardt MI, Ivan C, Ram PT, Wang Y, Baggerly K, Rodriguez-Aguayo C, Lopez-Berestein G, Yang SM, Maloney DJ, Yoshioka M, Strovel JW, Roszik J and Sood AK. 2018. Predicting novel therapies and targets: Regulation of Notch3 by the bromodomain protein BRD4. Molecular Cancer Therapeutics. DOI: 10.1158/1535-7163.MCT-18-0365

Wang S XZ, Hong Z, Jiao H, Zhu S, Zhao Y, Bi J, Qiu J, Zhang D, Yan J, Zhang L, Huang C, Li T, Liang L, Liao W, Ye Y and Ding Y. 2018. FOXF1 promotes angiogenesis and accelerates bevacizumab resistance in colorectal cancer by transcriptionally activating VEGFA. Cancer Letters 439:78-90. DOI: 10.1016/j.canlet.2018.09.026

Weiner H.L. ZD. 2000. Growth factor receptor tyrosine kinases: Cell adhesion kinase family suggests a novel signaling mechanism in cancer. Cancer Investigation 18:544-554. DOI: 10.3109/07357900009012194

Zhai J LR AN, Escalona R ,Tan FH ,Ratcliffe J ,Scoble JA ,Drummond CJ ,Tran N. 2018. PaclitaxelLoaded Self-Assembled Lipid Nanoparticles as Targeted Drug Delivery Systems for the Treatment of Aggressive Ovarian Cancer. ACS Applied Materials \& Interfaces. DOI: 10.1021/acsami.8b08125

\section{Figure and table legends}

Figure 1. Flow chart of study selection for GEO and TCGA based data.

Figure 2. Expression date in OC tissue from the GEO dataset.

(A)differential expression of FGFR1 in GSE105437; (B)differential expression of FGFR1 in GSE12470; (C)differential expression of FGFR1 in GSE12470 with subgroup analysis; (D)differential expression of EGFR in GSE105437; (E)differential expression of FGFR2 in GSE12470; E differential expression of FGFR3 in GSE12470.

Peer) reviewing PDF | (2020:05:48874:2:1:NEW 23 Dec 2020) 
430

431 Figure 3. ROC analysis of GSE105437 and GSE12470 of FGFR1 for the diagnosis of OC.

432 (A)GSE105437: The area under the ROC curve (AUC) 1.000, $\mathrm{p}=0.0009 ;$ (B)GSE12470: AUC 0.8256, $\mathrm{p}=0.0015$. 433

Figure 4. Meta-analysis of the combined SMD for FGFR1 expression between OC and normal groups in the GEO database using the fixed effects models.

436 (A)Forest plot; (B)funnel plot.

437

Figure 5. Differential expression of data between two sets of samples.

(A)GSE105437 (B)GSE12470. Red points represent upregulated genes (i.e. $|\mathrm{FC}|>2.0$ and a corrected P-value

of $<0.05$ ). Green points represent downregulated genes (i.e. $|\mathrm{FC}|>2.0$ and a corrected P-value of $<0.05$ ). Black points represent genes with no significant difference in expression. FC is the fold change.

Figure 6. Enrichment plots from gene set enrichment analysis (GSEA).

Common pathways in the GSE105437 (A) and GSE12470 (B) datasets according to GSEA, specific pathways involved FGFR1 GSE105437 datasets according to GSEA (C). ES, enrichment score; NES, normalized ES; NOM p-value, normalized p-value.

Figure 7. Kaplan-Meier estimates of OC overall survival of by residual tumor, stage and expression of FGFR1 (A) Residual tumor, $\mathrm{p}=0.001$; (B) Stage, $\mathrm{p}=0.148$; (C) Expression of FGFR1, $\mathrm{p}=0.233$.

Table 1:

Correlations between the relative expression of FGFR1 mRNA in OC tissues and patient clinical indicators 
Figure 1

Flow chart of study selection for GEO and TCGA based data.

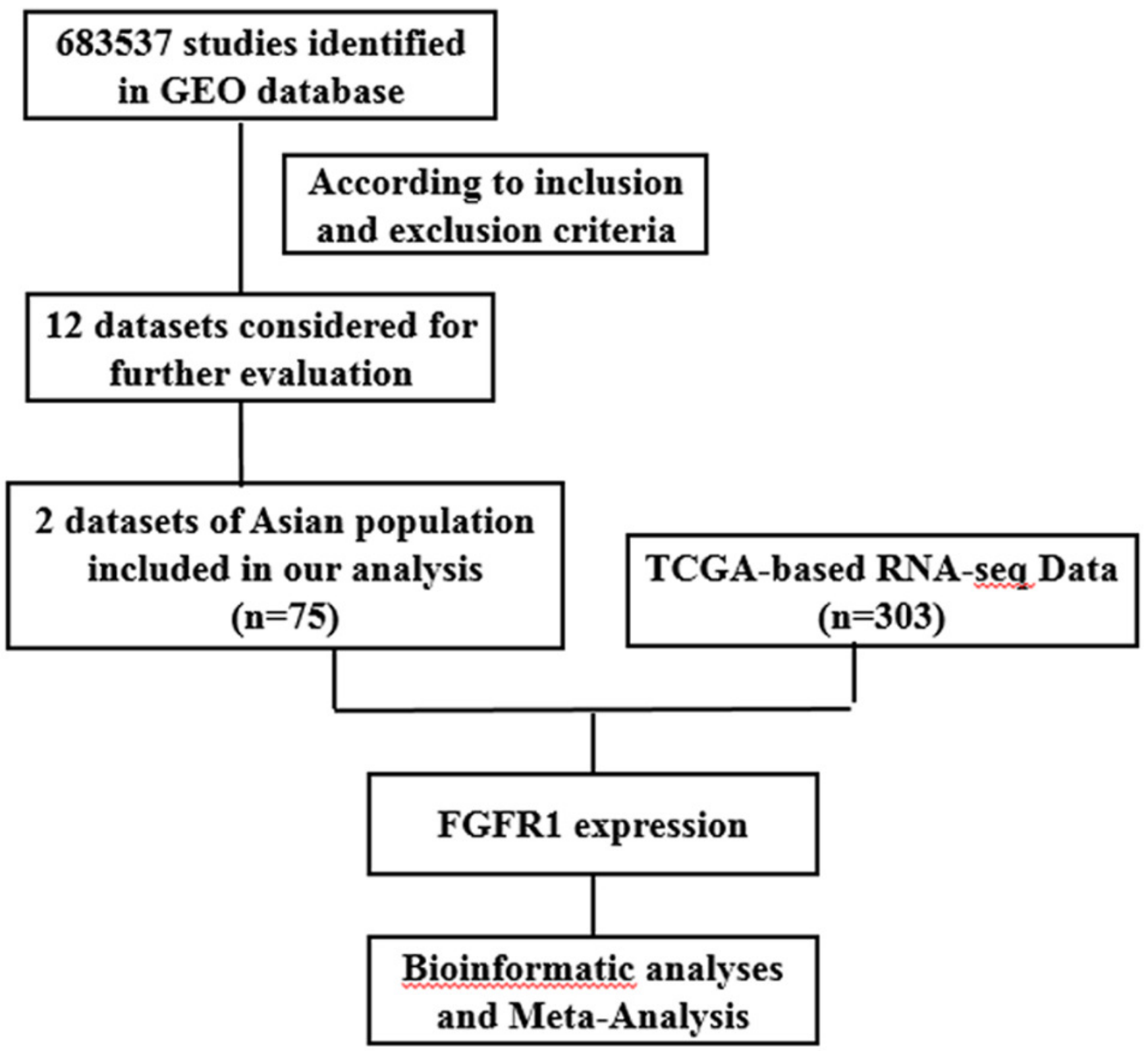


Figure 2

Expression date in OC tissue from the GEO dataset.

(A)differential expression of FGFR1 in GSE105437; (B)differential expression of FGFR1 in GSE12470; (C)differential expression of FGFR1 in GSE12470 with subgroup analysis;

(D)differential expression of EGFR in GSE105437; (E)differential expression of FGFR2 in GSE12470; E differential expression of FGFR3 in GSE12470.

A

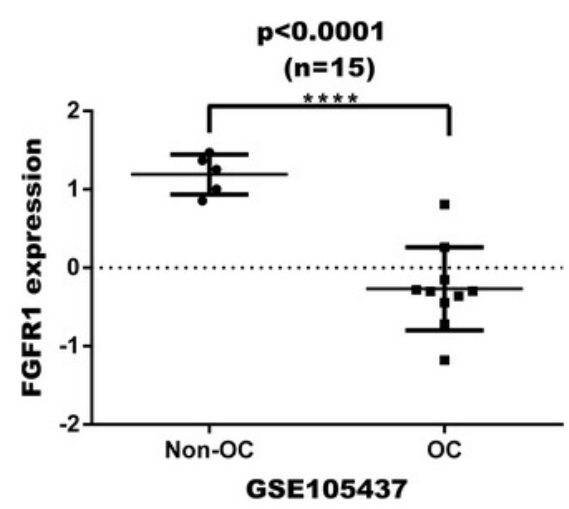

D

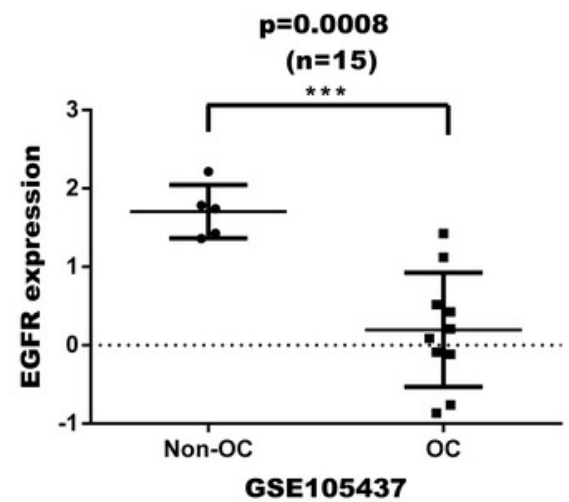

B

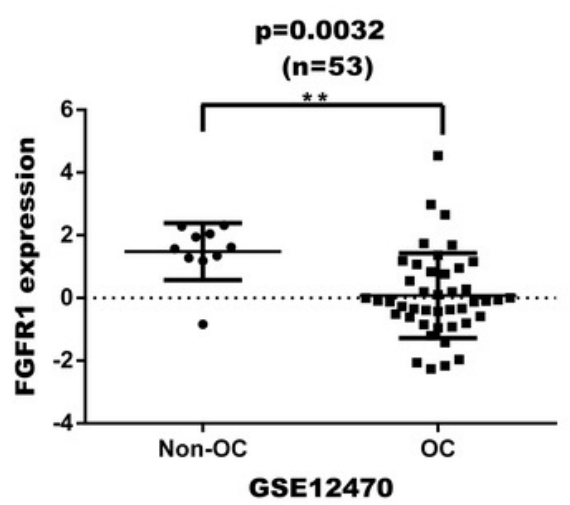

$\mathbf{E}$

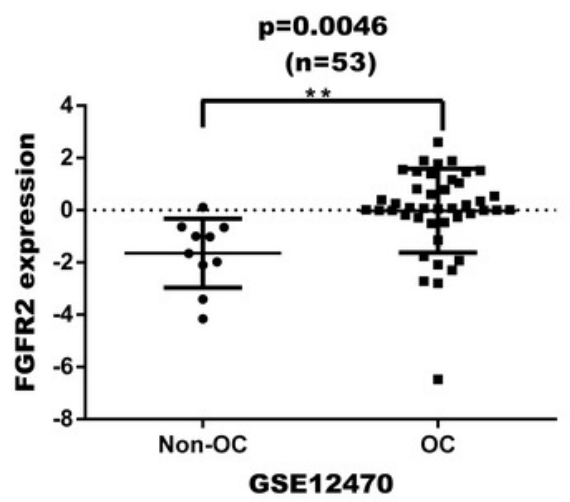

C

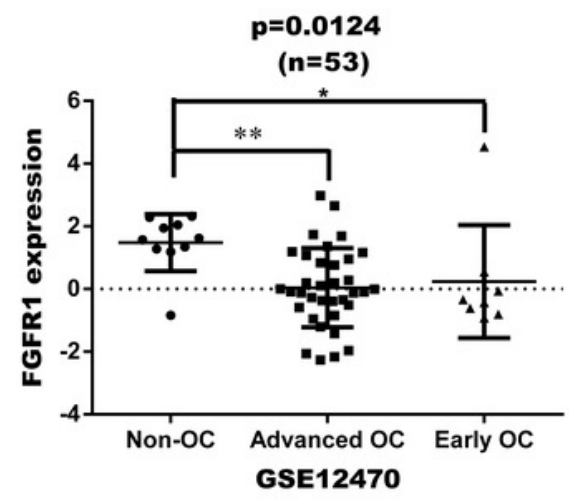

$\mathbf{F}$

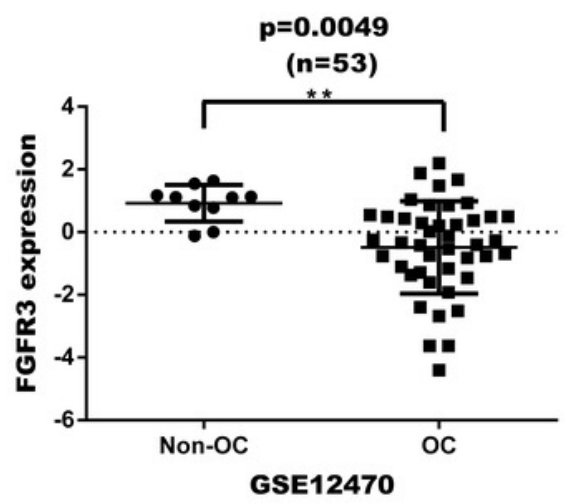


Figure 3

ROC analysis of GSE105437 and GSE12470 of FGFR1 for the diagnosis of OC.

(A)GSE105437: The area under the ROC curve (AUC) 1.000, $p=0.0009$; (B)GSE12470: AUC $0.8256, p=0.0015$.

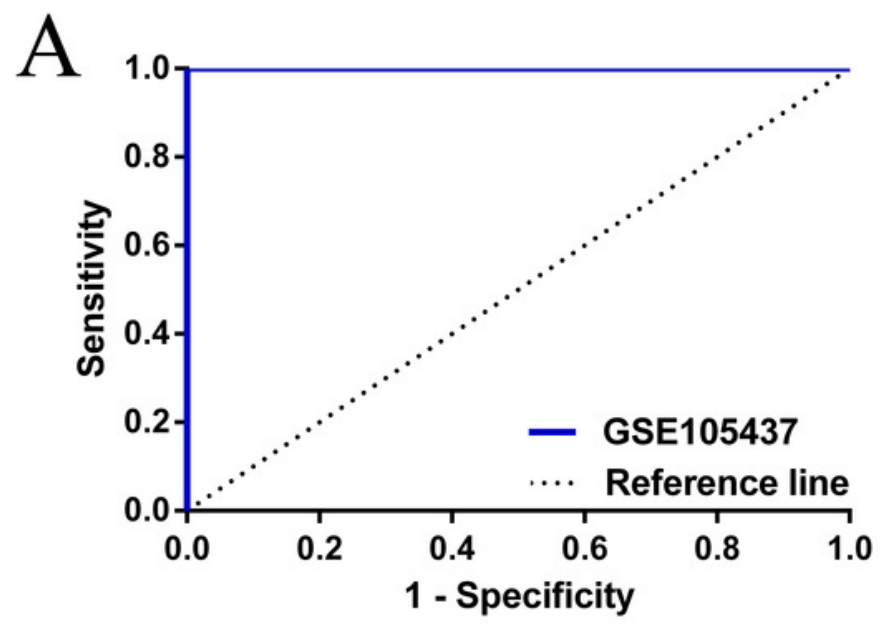

B

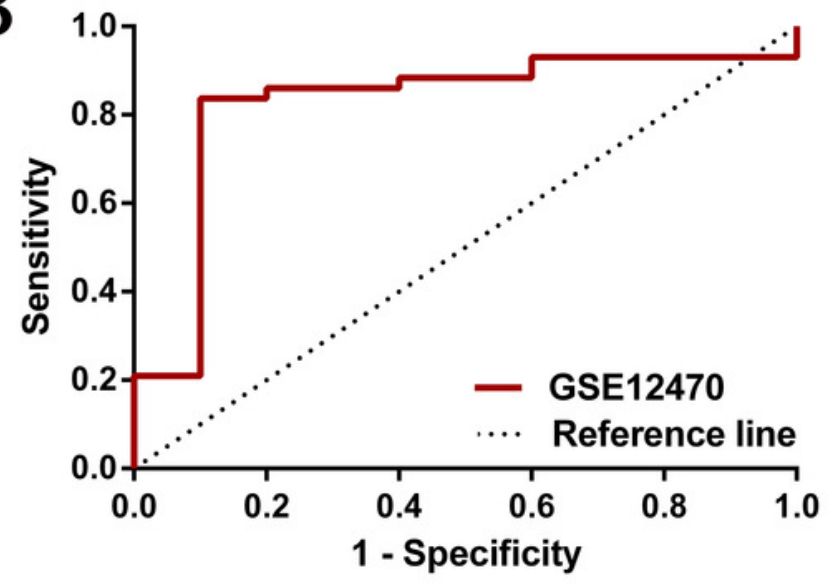


Figure 4

Meta-analysis of the combined SMD for FGFR1 expression between OC and normal groups in the GEO database using the fixed effects models.

(A)Forest plot; (B)funnel plot.

A

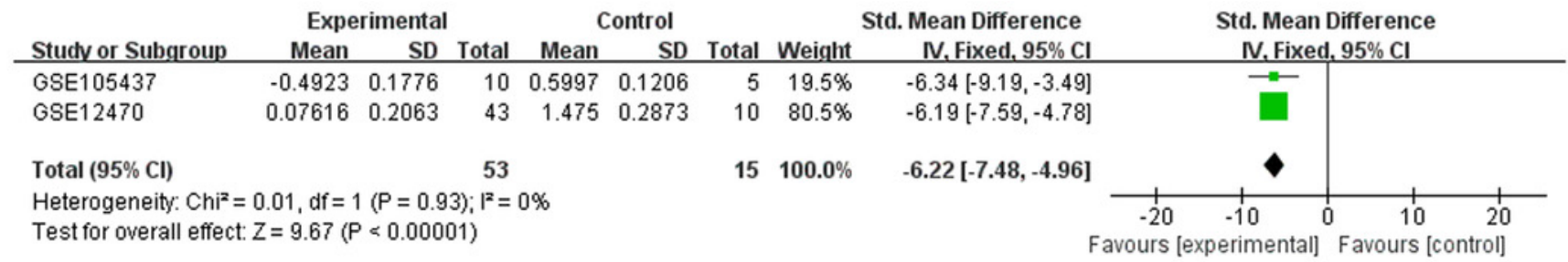

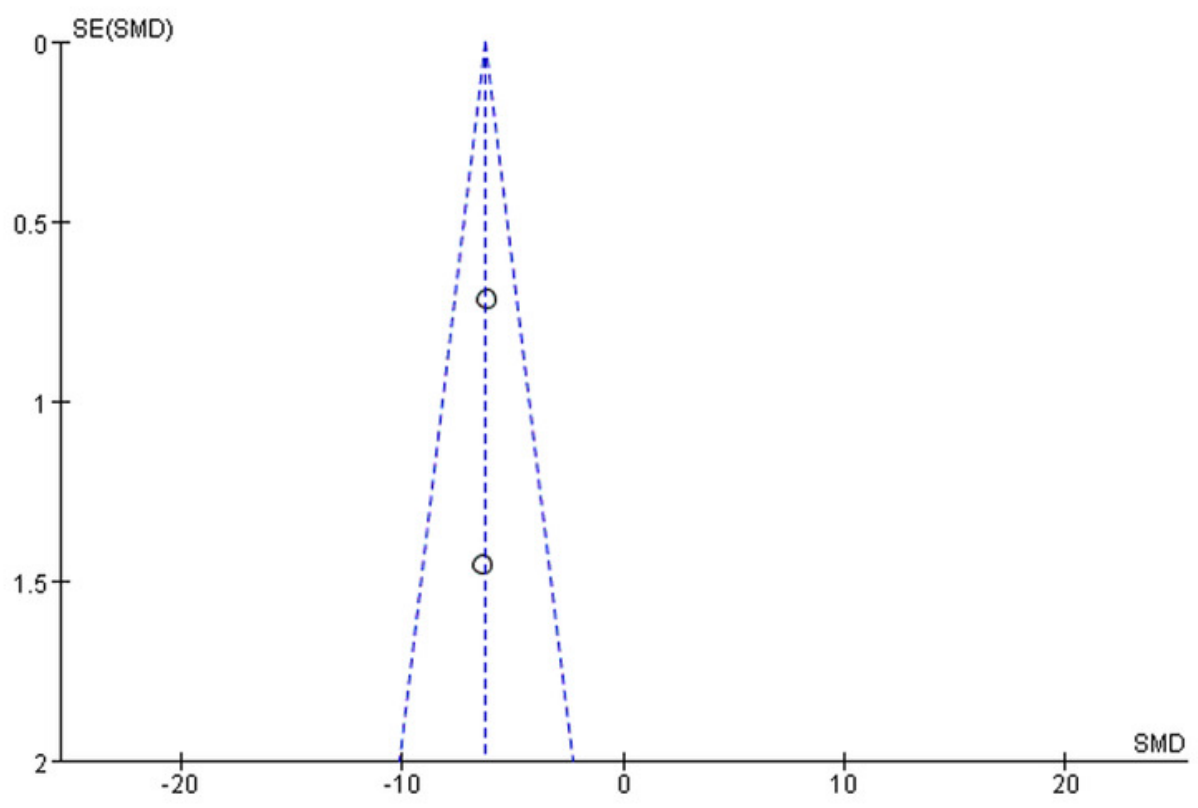




\section{Figure 5}

Differential expression of data between two sets of samples.

(A)GSE105437 (B)GSE12470. Red points represent upregulated genes (i.e. $|F C| \& \gamma \tau ; 2.0$ and a corrected P-value of $\& \lambda \tau ;$; 0.05). Green points represent downregulated genes(i.e. $|F C| \& \gamma \tau$; 2.0 and a corrected P-value of $\& \lambda \tau ; 0.05)$. Black points represent genes with no significant difference in expression. $\mathrm{FC}$ is the fold change.

A

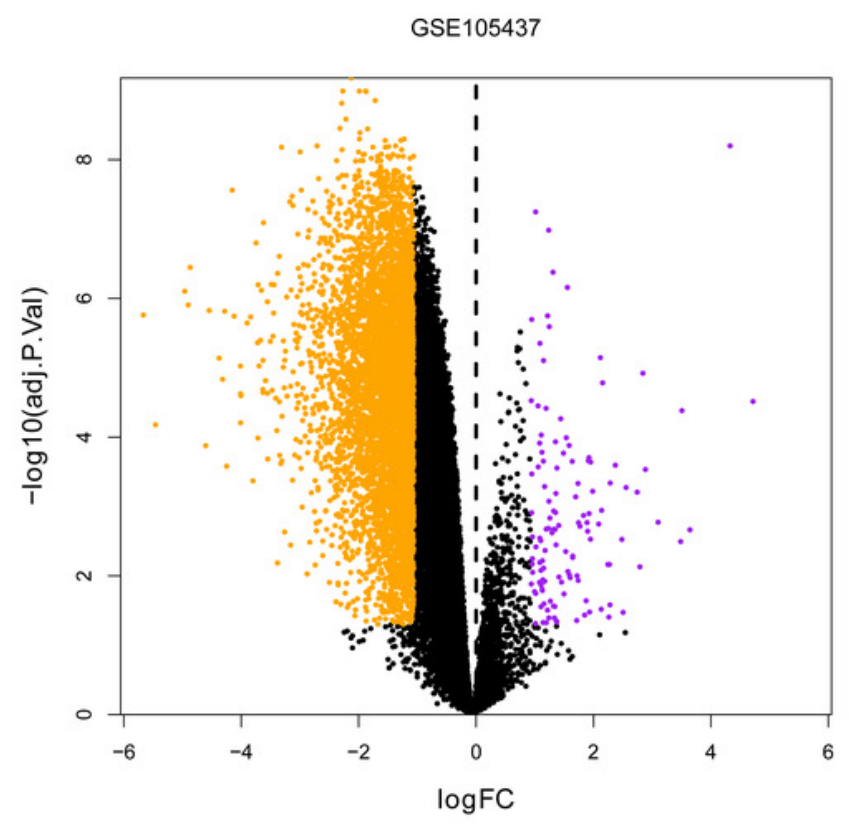

B

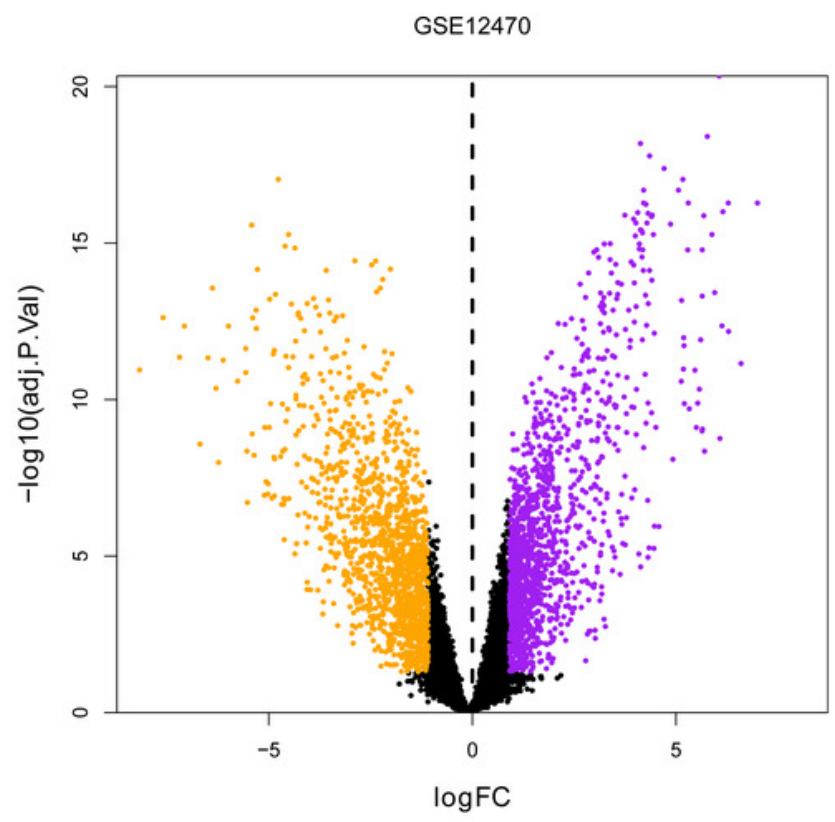


Figure 6

Enrichment plots from gene set enrichment analysis (GSEA).

Common pathways in the GSE105437 (A) and GSE12470 (B) datasets according to GSEA, specific pathways involved FGFR1 GSE105437 datasets according to GSEA (C). ES, enrichment score; NES, normalized ES; NOM p-val, normalized p-value. 


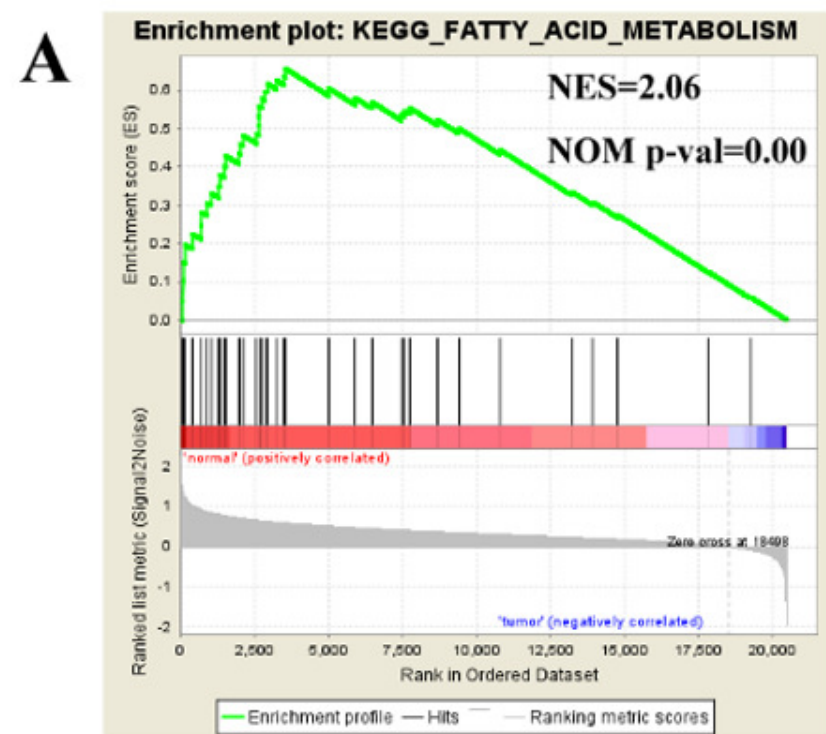

B
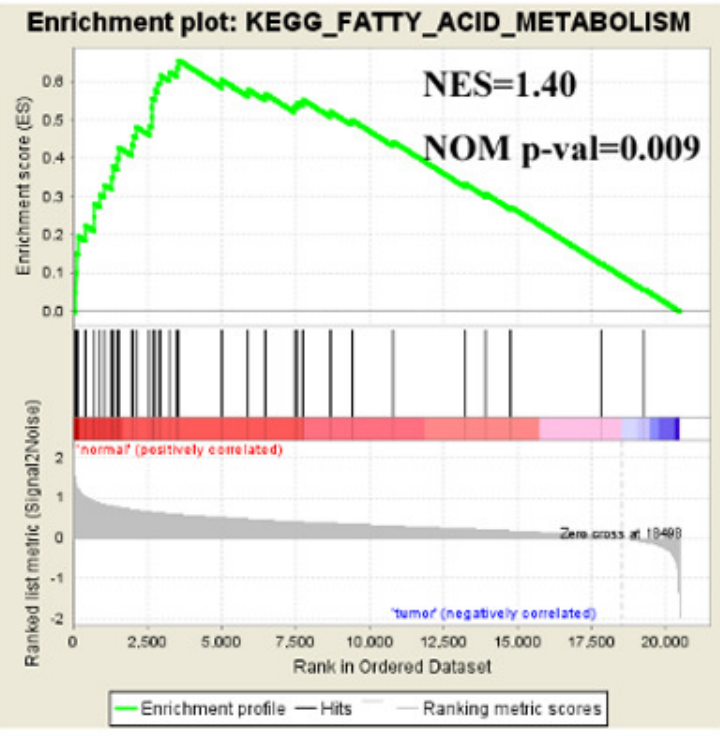

Enrichment plot: KEGG_ADHERENS_JUNCTION

C

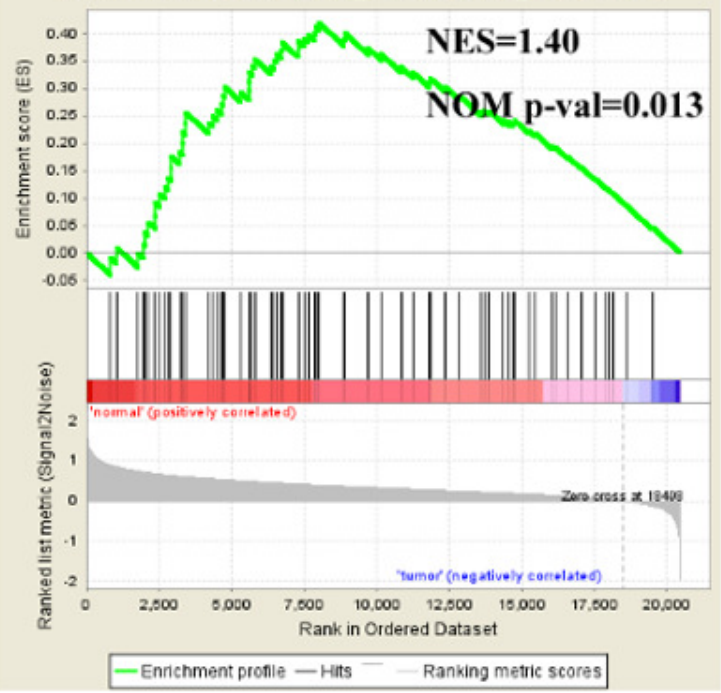

Enrichment plot: KRAS.KIDNEY_UP.V1_UP
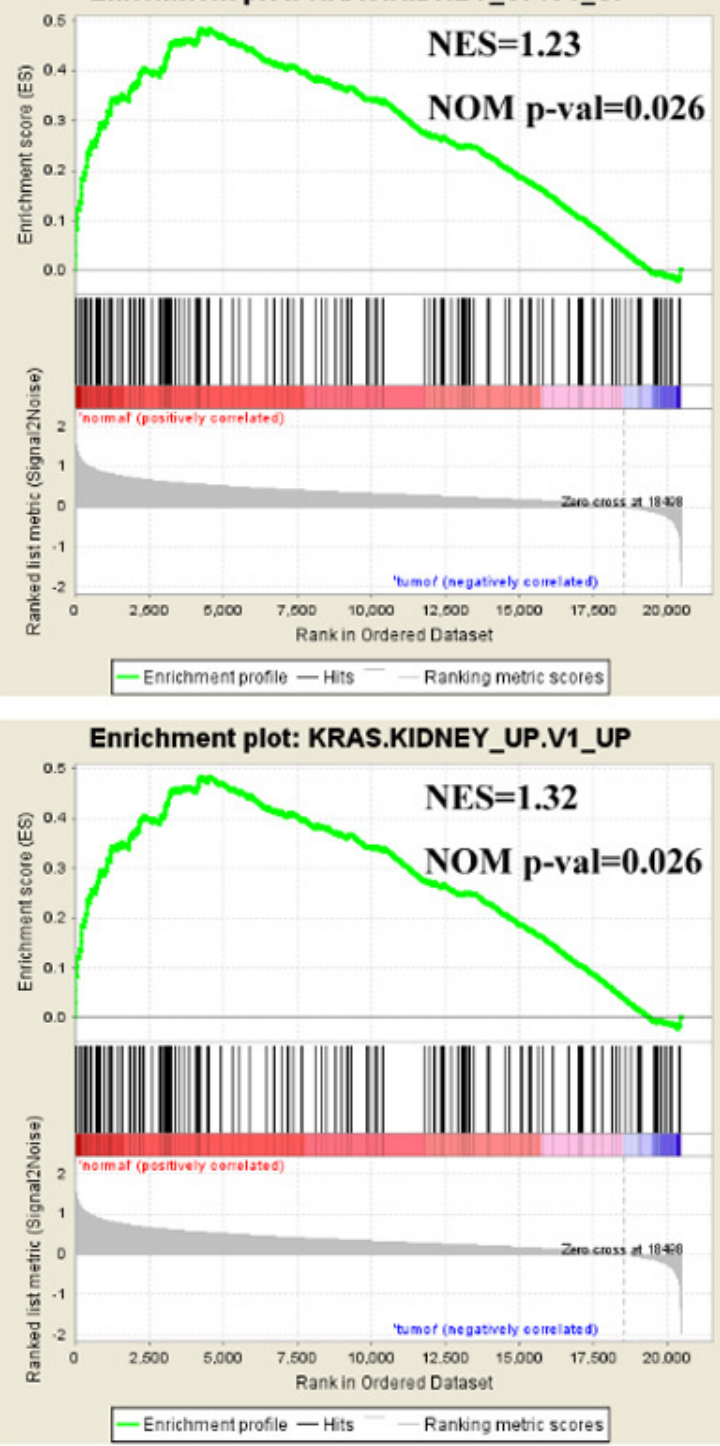
Figure 7

Kaplan-Meier estimates of OC overall survival of by residual tumor, stage and expression of FGFR1

$<$ !--[if !supportLists]-->(A) <!--[endif]-->Residual tumor, $p=0.001 ;$ (B) Stage, $p=0.148$; (C) Expression of FGFR1, $p=0.233$.

A

Survival according to Residuals

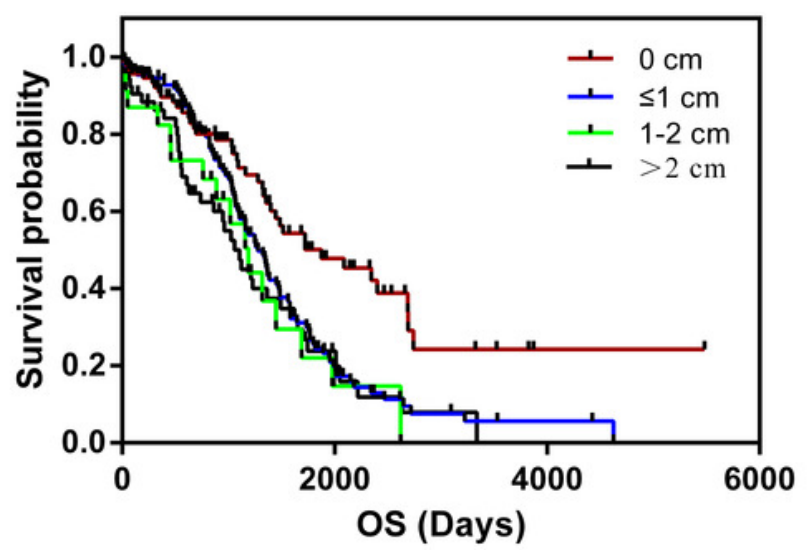

B

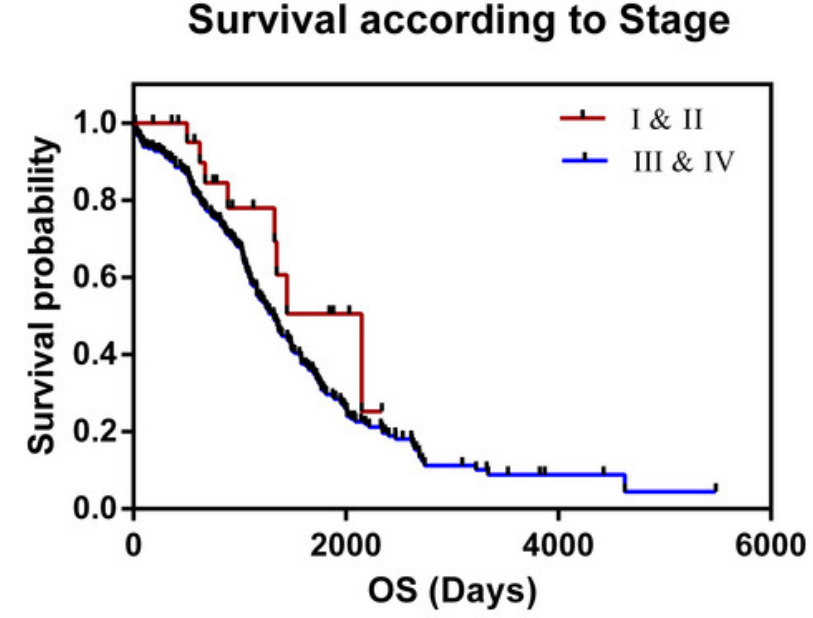

C

Survival according to FGFR1

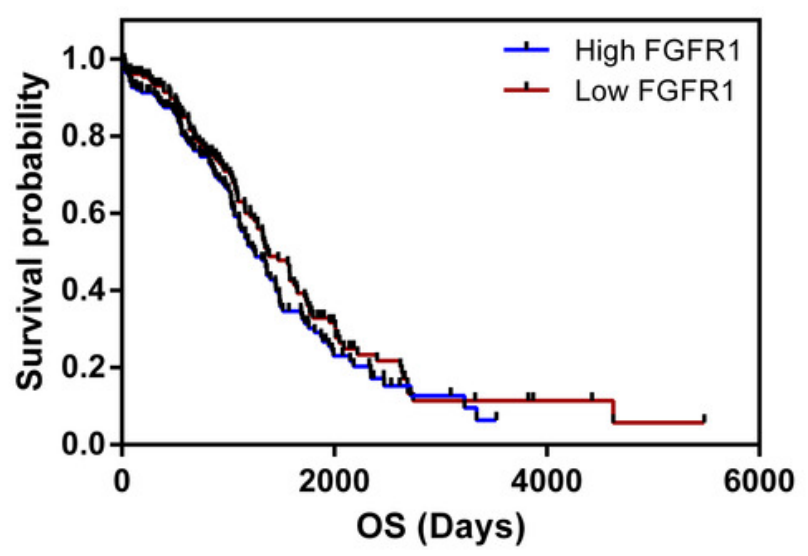




\section{Table $\mathbf{1}$ (on next page)}

Correlations between the relative expression of FGFR1 mRNA in OC tissues and patient clinical indicators 
1 Table 1:

2 Correlations between the relative expression of FGFR1 mRNA in OC tissues and patient clinical indicators 3

\begin{tabular}{|c|c|c|c|}
\hline $\begin{array}{c}\text { Clinicopathological } \\
\text { parameters }\end{array}$ & $\mathrm{N}$ & $\begin{array}{c}\text { Relative expression } \\
\text { of FGFR1 mRNA }\end{array}$ & $P$ value \\
\hline \multicolumn{4}{|l|}{ Age } \\
\hline$\leq 40$ & 10 & $11.45 \pm 0.3398$ & 0.4750 \\
\hline $40-60$ & 157 & $11.60 \pm 0.0887$ & \\
\hline$\geq 60$ & 136 & $11.75 \pm 0.1039$ & \\
\hline \multicolumn{4}{|l|}{ Stage } \\
\hline$|\&| \mid$ & 24 & $11.59 \pm 0.2201$ & 0.7667 \\
\hline III \& IV & 279 & $11.67 \pm 0.06965$ & \\
\hline \multicolumn{4}{|l|}{ Grade } \\
\hline $\mathrm{G} 1+\mathrm{G} 2$ & 34 & $11.59 \pm 0.1480$ & 0.8451 \\
\hline $\mathrm{G} 3+\mathrm{G} 4$ & 261 & $11.67 \pm 0.0741$ & \\
\hline Gx & 8 & $11.94 \pm 0.3241$ & \\
\hline \multicolumn{4}{|l|}{ Longest dimension } \\
\hline$\leq 1 \mathrm{~cm}$ & 92 & $11.60 \pm 0.1201$ & 0.2510 \\
\hline $1-2 \mathrm{~cm}$ & 169 & $11.63 \pm 0.0881$ & \\
\hline$\geq 2 \mathrm{~cm}$ & 42 & $11.93 \pm 0.1843$ & \\
\hline \multicolumn{4}{|l|}{ ANS } \\
\hline Bilateral & 209 & $11.63 \pm 0.0783$ & 0.5104 \\
\hline Unilateral & 94 & $11.73 \pm 0.1246$ & \\
\hline \multicolumn{4}{|l|}{ Residual tumor } \\
\hline 0 & 95 & $11.72 \pm 0.1156$ & 0.6568 \\
\hline$\leq 1 \mathrm{~cm}$ & 133 & $11.59 \pm 0.0973$ & \\
\hline $1-2 \mathrm{~cm}$ & 23 & $11.88 \pm 0.2868$ & \\
\hline $2 \mathrm{~cm}$ & 52 & $11.62 \pm 0.1671$ & \\
\hline
\end{tabular}




\section{Table 2 (on next page)}

Univariate analyse of survival of 303 OC patients ( using the Kaplan-Meier method ) 
1 Table 2:

2 Univariate analyses of survival of 303 OC patients (using the Kaplan-Meier method)

3

\begin{tabular}{|c|c|c|c|c|}
\hline Factor & $\mathrm{n}$ & $\begin{array}{l}\text { 3-year survival } \\
(\%)\end{array}$ & $X^{2}$ & $\mathrm{P}$ \\
\hline Age(years) & & & 6.463 & 0.04 \\
\hline$\leq 40$ & 10 & 71.4 & & \\
\hline $40-60$ & 157 & 70.5 & & \\
\hline$\geq 60$ & 136 & 51.2 & & \\
\hline Stage & & & 2.088 & 0.148 \\
\hline I \& II & 24 & 77.9 & & \\
\hline III \& IV & 279 & 60.9 & & \\
\hline Grade & & & 2.547 & 0.28 \\
\hline $\mathrm{G} 1+\mathrm{G} 2$ & 34 & 78.1 & & \\
\hline $\mathrm{G} 3+\mathrm{G} 4$ & 261 & 59.0 & & \\
\hline Gx & 8 & 87.5 & & \\
\hline $\begin{array}{l}\text { Longest } \\
\text { dimension }\end{array}$ & & & 0.47 & 0.79 \\
\hline$\leq 1 \mathrm{~cm}$ & 92 & 59.7 & & \\
\hline $1-2 \mathrm{~cm}$ & 169 & 65.4 & & \\
\hline$\geq 2 \mathrm{~cm}$ & 42 & 51.8 & & \\
\hline ANS & & & 0.574 & 0.449 \\
\hline Bilateral & 226 & 62.7 & & \\
\hline Unilateral & 77 & 58.3 & & \\
\hline $\begin{array}{l}\text { Residual } \\
\text { tumor }\end{array}$ & & & 16.670 & 0.001 \\
\hline 0 & 95 & 74.9 & & \\
\hline$\leq 1 \mathrm{~cm}$ & 133 & 61.1 & & \\
\hline $1-2 \mathrm{~cm}$ & 23 & 50.5 & & \\
\hline $2 \mathrm{~cm}$ & 52 & & & \\
\hline
\end{tabular}


50.0

FGFR1

1.425

0.233

\begin{tabular}{lcc}
$\begin{array}{l}\text { High } \\
\text { expression }\end{array}$ & 152 & 58.2 \\
$\begin{array}{l}\text { Low } \\
\text { expression }\end{array}$ & 151 & 65 \\
\hline
\end{tabular}

4

5

6

7

8

9

10

11 